\title{
Decoy receptor 3 alleviates hepatic fibrosis through suppressing inflammation activated by NF-KB signaling pathway
}

\author{
Zhenjing Jin ${ }^{A, E}$, Siqi Liu ${ }^{B, C}$, Qian Zhang ${ }^{D, E}$, Xue Shao ${ }^{B, F}$, Jingting Ma ${ }^{C}$, Liulan Pan ${ }^{D-F}$ \\ The Second Clinical Hospital, Jilin University, Changchun, China \\ A - research concept and design; $\mathrm{B}$ - collection and/or assembly of data; $\mathrm{C}$ - data analysis and interpretation; \\ $D$ - writing the article; $E$ - critical revision of the article; $F$ - final approval of the article
}

Address for correspondence

Liulan Pan

E-mail: woshipanliulan@126.com

Funding sources

None declared

Conflict of interest

None declared

Received on 0ctober 19, 2016

Reviewed on November 5,2016

Accepted on January 10, 2017

DOI

\begin{abstract}
Background. Hepatic fibrosis is a reversible pathological process. Inflammatory responses are the prevailing reactions during hepatic fibrosis. Decoy receptor 3 (DcR3) has been reported to have an anti-inflammatory effect.
\end{abstract}

Objectives. The aim of the study was to investigate the preventive effects of DcR3 on hepatic fibrosis.

Material and methods. Hepatic fibrosis was induced in rats by administering intraperitoneally (ip.) $1 \%$ dimethylnitrosamine (DMN). DCR3 plasmid was delivered into rats by intravenous injection. After 4 weeks, the expression of DCR3, TNF-like molecule $1 A$ (TL1A) and a-SMA of the liver tissue were checked. The levels of inflammatory cytokines such as TNF- $a, I L-6$ and IL-1 $\beta$ were detected using western blotting and quantitative real-time reverse transcription-polymerase chain reaction ( $q R T-P(R)$. Masson's trichrome staining for histopathological changes of the liver tissue was observed. Finally, the activity of NF-KB in the liver was examined by enzyme-linked immunosorbent assay (ELISA).

Results. A higher expression of DcR3 was observed in rats treated with $D c R 3(p<0.05)$. Histological results showed that DcR3 significantly attenuated pathology in hepatic fibrosis rats. Consistently, mRNA and protein levels of a-SMA, TL1A, TNF- $a$, IL-6, and IL-1 $\beta$ were repressed in the liver tissue after treatment with DCR3 $(p<0.05)$. Moreover, DcR3 also inhibited the activation of NF-kB in the liver tissue $(p<0.05)$.

Conclusions. This study demonstrated that DcR3 attenuated liver injury and inflammatory responses in rats with hepatic fibrosis. We suggest DcR3 may be a prophylactic and promising therapeutic agent in the treatment of hepatic fibrosis.

Key words: decoy receptor 3, hepatic fibrosis, inflammatory response, NF-kB, TNF-like molecule $1 \mathrm{~A}$

\section{$10.17219 /$ acem/68387}

\section{Copyright}

Copyright by Author(s)

This is an article distributed under the terms of the

Creative Commons Attribution Non-Commercial License

(http://creativecommons.org/licenses/by-nc-nd/4.0/) 


\section{Introduction}

Hepatic fibrosis is a wound-healing response to chronic liver injury. It is a reversible pathological process, but results in cirrhosis or liver failure if inappropriately treated. ${ }^{1}$ The pathological characteristic of hepatic fibrosis is sustained liver damage leading to chronic inflammation and liver death, followed by the release of cytokines by activated Kuppfer cells and endothelial cells, which leads to the formation of liver fibrosis by excessive accumulation of scar tissue and deposition of extracellular matrix (ECM) proteins as a result of further activation of hepatic stellate cells (HSCs). ${ }^{2-6}$ Therefore, inhibiting inflammation and liver cell apoptosis after liver damage is the key point to reverse liver fibrosis.

Decoy receptor 3 (DcR3), a soluble protein, belongs to the tumor necrosis factor receptor (TNFR) superfamily. ${ }^{7}$ DcR3 is found in humans but not in mice, and its encoding gene is located on human chromosome $20 \mathrm{q} 13.3 .^{8}$ DcR3 competitively interacts with its primary ligands, including Fas ligand (FASLG), T lymphocytes (LIGHT) and TNF-like molecule 1A (TL1A), to negatively regulate their ligandreceptor downstream signaling. ${ }^{9-11}$ It has been reported that DcR3 was overexpressed in various human cancers by negatively regulating Fas-mediated apoptosis. ${ }^{8,12}$ Moreover, growing evidence has demonstrated that DcR3 is involved in the regulation of immune responses by activating "reverse signaling" after binding with their receptors. ${ }^{13}$ Treatment with DcR3 protein reduces the levels of cytokines, including TNF- $\alpha$, IL- 6 and IFN- $\gamma$ in the blood and peritoneal lavage fluid of mice with sepsis. ${ }^{14}$ Another study discovered that DcR3 reduced the TLR2-induced cytokine production by B cells. ${ }^{15}$ All this evidence suggests that DcR3 plays an important role in the inflammatory response.

TL1A has been identified as a ligand of death receptor 3 (DR3) and DcR3. The major downstream signaling pathway of DR3/TL1A is the NF-kB pathway, which is involved in the treatment of inflammation by regulating the secretion of proinflammatory cytokines like TNF- $\alpha$, IL-6 and IL-1 $\beta$. More importantly, TL1A can promote the survival and secretion of proinflammatory cytokines by activating $\mathrm{T}$ cells in vitro. ${ }^{10} \mathrm{Shu}$ et el. found that the inhibition of MAPK and NF- $\mathrm{kB}$ signaling pathways alleviated carbon tetrachloride $\left(\mathrm{CCl}_{4}\right)$-induced liver fibrosis in mice with Toll-like receptor 5 (TLR5) deficiency. ${ }^{16}$ A proteome-wide quantitative phosphoproteomic analysis showed that DcR3 modulates the activity of key kinases critical for the activation of MAP kinases, and NF-kB activation. ${ }^{17}$ DcR3 can bind with TL1A by competing with DR3, but the role of DcR3 in the development of liver fibrosis is still unknown. Since DcR3 plays a pivotal role in the inflammatory response by binding to TL1A, we hypothesize that DcR3 may moderate liver fibrosis progress via attenuating inflammatory responses and liver injury.

DcR3 has been supposed to negatively regulate the DR3/
TL1A pathway by a competitive combination with TL1A. In the present study, we evaluated the role of the DcR3 gene in the process of $1 \%$ dimethylnitrosamine (DMN)stimulated fibrosis of liver in rats. Our results show that the DcR3 gene may be involved in the progress of fibrosis via the inhibition of NF-kB signaling pathways activated by the DR3/TL1A pathway.

\section{Material and methods}

\section{Establishment of hepatic fibrosis rat model}

Male Wistar rats (wild-type, WT), weighing 180-220 g, were purchased from the experimental animal center of Jilin University (China). All rats were housed in a temperature- $\left(25^{\circ} \mathrm{C}\right)$ and humidity-controlled environment with food and water provided in the cages. All rats were randomly divided into 3 groups as follows: 1 . the control group (WT group); 2 . the $1 \% \mathrm{DMN}+$ empty vector group (EV group); 3. the $1 \% \mathrm{DMN}+\mathrm{DcR} 3$ vector group (DcR3 group). DMN (Zhenzhun Ltd., Shanghai, China) was dissolved in saline and finally diluted to $1 \%$. After 1-week adaptation the EV and DcR3 group rats were administered by intraperitoneal (ip.) injection with $1 \mu \mathrm{L} / \mathrm{g}$ body weight $1 \%$ DMN 3 times every week for 4 weeks. Meanwhile, WT group rats were similarly injected with an equal volume of saline. The study was approved by the Ethics Committee on Animal Research at the Jilin University Animal Care and Use Committee (No. SYXK (Ji) 2008-0010/0011).

\section{Decoy receptor 3 gene therapy}

The pEF1 $\alpha$-IRES-DsRed-Express2-DcR3 was constructed as described previously. ${ }^{18}$ Briefly, the DcR3 gene was isolated by the real-time reverese transcription-polymerase chain reaction (RT-PCR) using the forward primer 5'GTCGACATGAGGGCGCTGGAGG3' and reverse primer 5'GGATCC TCAGTGCACAGGGAGGAA3'. The amplified product was cloned into pEF1a-IRES-DsRed-Express2 vector (Takara, Dalian, China) to produce the vectors pEF1 $\alpha$-IRES-DsRed-Express2-DcR3.

The DcR3 group rats were injected with DcR3 plasmid by tail intravenous (iv.) injection, while the EV group rats were similarly administered with an equal EV. All the rats were sacrificed 4 weeks after vector injection. The liver tissues were carefully harvested and stored appropriately until analysis.

\section{Histopathologic examination of liver tissues}

The liver tissues were fixed with 4 paraformaldehyde, cut into 4-5 $\mu \mathrm{m}$ thick sections and mounted on slides. Masson's trichrome staining was conducted to evaluate the pathological changes such as collagen deposition in the 
liver tissue. The sections were stained in iron hematoxylin solution for $10 \mathrm{~min}$ after dewaxing. After wash, the sections were stained in Biebrich scarlet-acid fuchsin solution for 10-15 min. Then, the slices were washed and differentiated in phosphomolybdic-phosphotungstic acid solution for 10-15 min. Next, the sections were transferred into aniline blue solution and stained for 5-10 $\mathrm{min}$, rinsed briefly in distilled water and differentiated in $1 \%$ acetic acid solution for 2-5 min. Finally, dehydration and mount were performed after wash. The method was described in the handbook of Trichrome Stain (Masson's) Kit (Sigma-Aldrich, St. Louis, USA). All the tissue sections were observed under a microscope.

\section{Determination of DcR3, TL1A, a-SMA, TNF- $\alpha$, IL- 6, IL-1 $\beta$, and $\beta$-actin in the liver by western blot analysis}

To detect the expression of DcR3, TL1A, $\alpha$-SMA, TNF- $\alpha$, IL-6, IL-1 $\beta$, and $\beta$-actin, total protein of the liver was extracted using RIPA buffer, then the nuclear and cytoplasmic proteins were extracted using a nuclear/cytoplasmic isolation kit (Takara, Dalian, China). The protein concentration was determined by a BCA Protein Assay Kit (Takara, Dalian, China). The protein was separated by $15 \%$ SDS-PAGE and transferred onto a PVDE membrane (BioRad Laboratories Inc., Hercules, USA). The membrane was blocked with $5 \%$ skim milk powder for $2 \mathrm{~h}$ and incubated overnight with the primary antibody. Each membrane was washed and incubated with the secondary antibodies for $1.5 \mathrm{~h}$. The immunoblots were developed using an ECL Advanced Western Blotting Detection Kit (Invitrogen, Carlsbad, USA).

\section{Determination of DcR3, TL1A, $a$-SMA, TNF- $\alpha$, IL- 6, IL-1 $\beta$, and $\beta$-actin in the liver by real-time reverse transcription- polymerase chain reaction (RT-PCR)}

To evaluate the mRNA transcriptional levels of DcR3, TL1A, $\alpha$-SMA, TNF- $\alpha$, IL- 6 , and IL- $1 \beta$ in the livers of each group, the total RNA was isolated using trizol reagent ( $\mathrm{Ta}$ kara, Dalian, China) according to the manufacturer's instructions. Then, total RNA was reverse-transcribed to cDNA using the reverse transcription kit (Takara Biological Company, Dalian, Japan). RT-PCR was performed using Eppendorf AG-5341 fluorescence quantitative instrument. The procedure of qPCR was administered as following: $95^{\circ} \mathrm{C}$ for $3 \mathrm{~min}$; 35 cycles of $94^{\circ} \mathrm{C}$ for $30 \mathrm{~s}, 58^{\circ} \mathrm{C}$ for $30 \mathrm{~s}$ and $72^{\circ} \mathrm{C}$ for $3 \mathrm{~min}$; and a final extension period at $72^{\circ} \mathrm{C}$ for $10 \mathrm{~min}$. All primers were synthesized by Shanghai Sangon (Shanghai, China) (Table 1). The results were calculated using the $2^{-\Delta \Delta C t}$ method, and the gene GAPDH was used as an internal control.
Table 1. Primer nucleotide sequence of the liver tissue DCR3, TL1A, $a-S M A$, TNF- $a, I L-6, I L-1 \beta$, and $\beta$-actin

\begin{tabular}{|c|c|c|}
\hline Genes & Nucleotide sequence of primer $\left(5^{\prime}-3^{\prime}\right)$ & Product size (bp) \\
\hline \multirow{2}{*}{ DCR3 } & F:\#CGCTGGTTTCTGCTTGGAG & \multirow{2}{*}{122} \\
\hline & R:\#AGCTGCTGGCTGAGAAGGTG & \\
\hline \multirow{2}{*}{$T L 1 A$} & F:\#TCTACTCCCAGATCACATTCCG & \multirow{2}{*}{180} \\
\hline & R:\#ACCAGTTGCTGCTTATTTCACAC & \\
\hline \multirow{2}{*}{$a-S M A$} & F:\#AGGAGGATTCCGTGCTGTTC & \multirow{2}{*}{312} \\
\hline & R:\#TGGGCTTGATGTTATCTGATTT & \\
\hline \multirow{2}{*}{ TNF-a } & F:\#CCCCTTTATCGTCTACTCCTC & \multirow{2}{*}{134} \\
\hline & R:\#TTCAGCGTCTCGTGTGTTTC & \\
\hline \multirow{2}{*}{$\| L-6$} & F:\#CTTCGGTCCAGTTGCCTTCT & \multirow{2}{*}{228} \\
\hline & R:\#GCCTCTTTGCTGCTTTCACA & \\
\hline \multirow{2}{*}{$I L-1 \beta$} & F:\#TTACAGTGGCAATGAGGATG & \multirow{2}{*}{131} \\
\hline & R:\#TGTAGTGGTGGTCGGAGATT & \\
\hline \multirow{2}{*}{$\beta$-actin } & F:\#CGGCTACAGCTTCACCACCA & \multirow{2}{*}{143} \\
\hline & R:\#CGGGCAGCTCGTAGCTCTTC & \\
\hline
\end{tabular}

DCR3 - decoy receptor 3; TL1A - TNF-like molecule 1A; $a$-SMA - smooth muscle actin; TNF- $a$ - tumor necrosis factor; IL-6 - interleukin 6; $I L-1 \beta$ - interkeukin 1 beta; $F$ - forward primer; $R$ - reverse primer.

\section{Expression of NF-kB by enzyme-linked immunosorbent assay (ELISA)}

The production levels of NF- $\mathrm{KB}$ in the liver were assayed by enzyme-linked immunosorbent assay (ELISA) kit (Takara, Dalian, China) according to the manufacturer's protocols.

\section{Statistical analysis and software}

The data was analyzed by SPSS 19.0 software, and comparisons between multiple groups were performed by a one-way analysis of variance (ANOVA) followed by the Dunnett multiple comparison tests to determine statistical significance. The data was expressed as mean $\pm \mathrm{SD}$ (standard deviation) and a p-value of $<0.05$ was considered statistically significant. The homology analysis was performed by BLAST and the structure of protein was predicted by SMART online tool.

\section{Results}

\section{DCR3 decreased the inflammatory cytokines levels by suppressing the NF-KB signaling pathway}

Inflammation of the liver was reported to contribute to the development of liver fibrosis. This study aimed to investigate the effect of DcR3 on inflammatory cytokines in hepatic fibrosis, and, therefore, the TNF- $\alpha$, IL- 6 and IL-1 $\beta$ levels were detected in the liver. The results showed that the TNF- $\alpha$, IL- 6 and IL- $1 \beta$ mRNA levels in the liver 

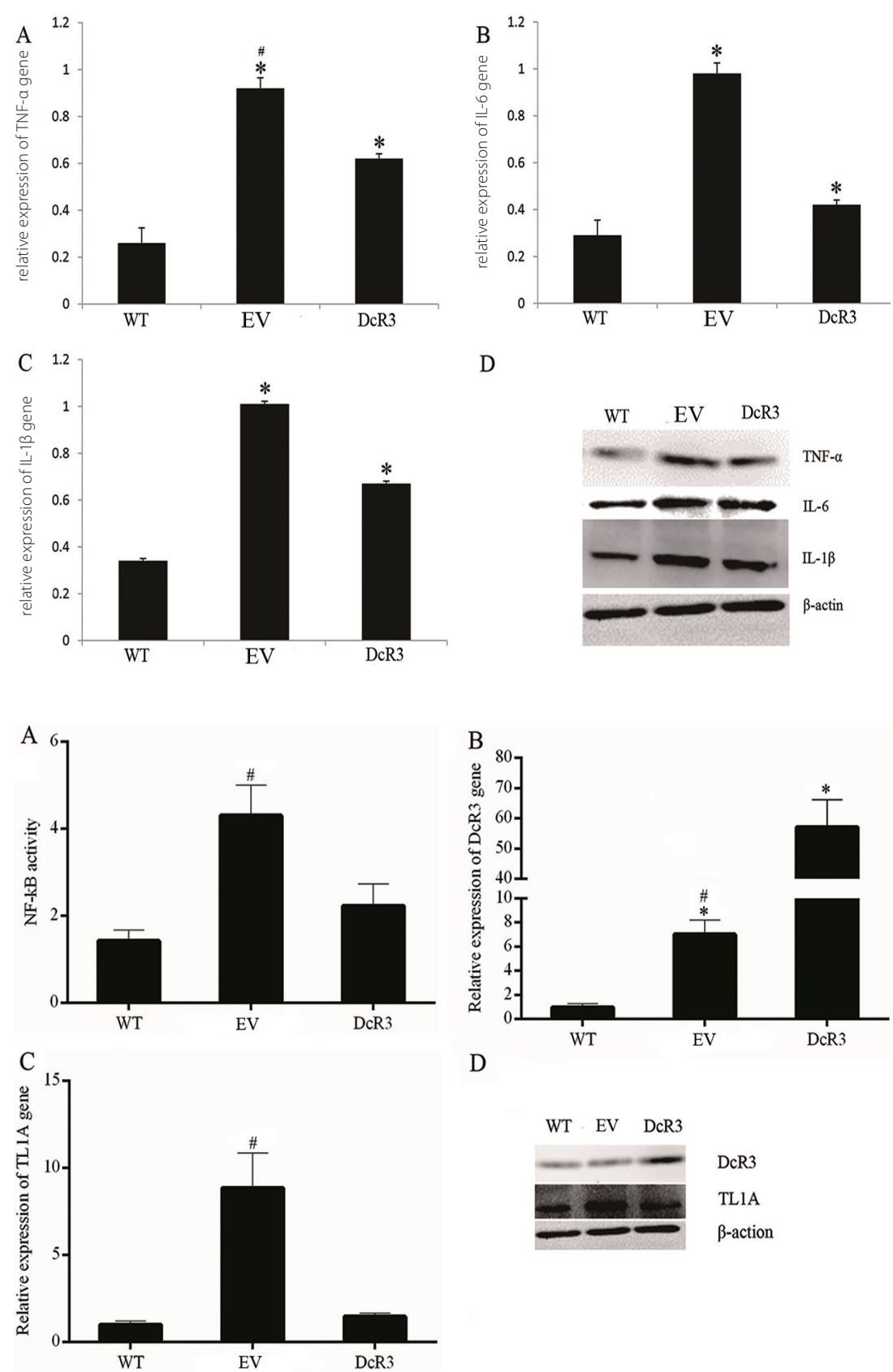

$\mathrm{D}$

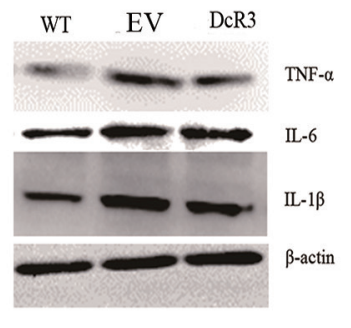

Fig. 1. DcR3 decreased the inflammatory cytokines levels in the liver tissue of 1\% DMN-induced hepatic fibrosis rats. A, B and C - analysis of the TNF- $a, I L-6$ and IL-1 $\beta$ mRNA expression by qRT-PCR, respectively. $D$ - analysis of the TNF- $a, I L-6$ and $I L-1 \beta$ protein expression by western blot, respectively. Data is shown as mean $\pm S D$ of 10 rats in each group

WT - control group (wild-type); EV - 1\% DMN + empty vector group; DcR3 - 1\% DMN + DcR3 vector group; ${ }^{*} \mathrm{p}<0.05$ compared with WT group; ${ }^{\mathrm{p}}<0.05$ compared with EV group; DcR3 - decoy receptor 3; DMN - dimethylnitrosamine.
Fig. 2. DCR3 decreased the TL1A levels and inhibited NF-KB activation in the liver tissue of $1 \%$ DMNinduced hepatic fibrosis rats. A - analysis of NF-KB activity by ELISA. B and C - analysis of the DCR3 and TL1A mRNA expression by qRT-PCR, respectively. $D$ - analysis of the DCR3 and TL1A protein expression by western blot, respectively. Data is shown as mean \pm SD of 10 rats in each group

WT - control group (wild-type); EV - 1\% DMN + empty vector group; DcR3 - 1\% DMN + DcR3 vector group; ${ }^{*} p<0.05$ compared with WT group; ${ }^{*} p<0.05$ compared with EV group; DCR3 - decoy receptor 3; TL1A - TNF-like molecule 1A; ELISA - enzyme-linked immunosorbent assay; DMN - dimethylnitrosamine. dramatically decreased in the DcR3 group compared with the EV group $(\mathrm{p}<0.05)$ (Fig. $1 \mathrm{~A}-\mathrm{C})$. In addition, western blot analysis also showed that rats treated with DcR3 exhibited lower TNF- $\alpha$, IL- 6 and IL- $1 \beta$ protein levels than the EV group (Fig. 1D). These results indicated that DcR3 alleviated the $1 \% \mathrm{DMN}$-induced inflammatory responses in rats.

We found that DcR3 could inhibit the expression of proinflammatory cytokines, and then we further studied whether DcR3-mediated protection against liver fibrosis was associated with alterations in NF- $\mathrm{kB}$ activation. ELISA tests showed that rats treated with DcR3 secreted lower NF-кB levels compared with EV rats (p < 0.05) (Fig. 2D). On the basis of these results, we suggest that DcR3 inhibited the TNF- $\alpha$, IL- 6 and IL- $1 \beta$ production through suppressing the NF-kB signaling pathway.

\section{DcR3 decreased the inflammatory cytokines levels by a combination with TL1A}

TL1A was recognized as a ligand of DR3 and DcR3 which negatively regulated the downstream signaling cascades of DR3/TL1A such as NF-kB activation. Therefore, we next wondered whether DcR3 inhibited the production of inflammatory cytokines by binding with TL1A. First, we validated the DcR3 overexpression in hepatic fibrosis rats by western blot analysis and RT-PCR. The results showed that DcR3 increased after injection with DcR3 vector $(\mathrm{p}<0.05)$ (Fig. 2A, C). We also examined the expression of TL1A in hepatic fibrosis rats. TL1A protein and mRNA expression was obviously decreased in the DcR3 group compared with the EV group ( $<<0.05$ ) (Fig. 2B, C). Our results suggest that DcR3 might interact with TL1A to 
negatively regulate the downstream signaling cascades of DR3/TL1A.

\section{DcR3 inhibited the inflammatory cell infiltration and collagen accumulation in hepatic fibrosis rats}

Next, we evaluated the morphological characteristics of the rat livers by Masson's trichromic staining. No pathological changes or immune reactions were observed in the liver tissue in the WT group. However, the cell swelling, fatty infiltration, extensive accumulation of collagen, cell death, and leukocyte infiltration became overt in the liver tissue in the EV group (Fig. 3A), whereas DcR3 markedly attenuated these pathological changes (Fig. 3A). These results indicate that hepatic fibrosis rats had marked fibrotic changes in the livers after the treatment with DcR3.

\section{DcR3 inhibited the expression of a-SMA in the rat livers}

The expression of $\alpha$-SMA is associated with the activity of HSCs and the degree of hepatic fibrosis. Western blot analysis and RT-PCR showed a great decrease in the $\alpha$-SMA expression in the DcR3 group compared with the EV group (Fig. 3B, C). Combined with histological observation, our results suggest that DcR3 can alleviate hepatic fibrosis induced by $1 \% \mathrm{DMN}$.

\section{Homology analysis and the prediction of DcR3 domain}

We predicted the DcR3 domain structure and analyzed the homology in humans and rats by online software. The DcR3 protein has 4 TNF receptor repeats in extracellular domain (Fig. 4A). The structure of the DcR3 protein is quite similar to rat TNFR1- $\alpha$, and a homology analysis indicates high conservation between DcR3 and TNFR1- $\alpha$ in rats and humans (Fig. 4B).

A

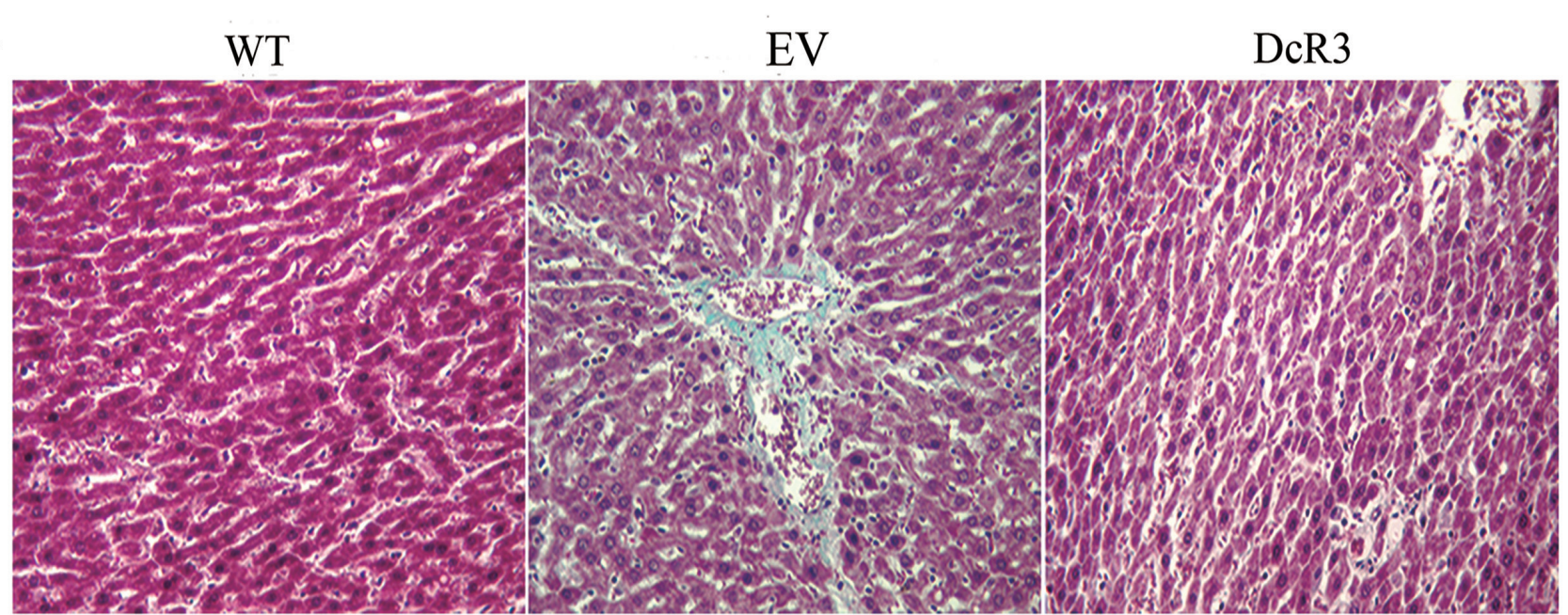

B

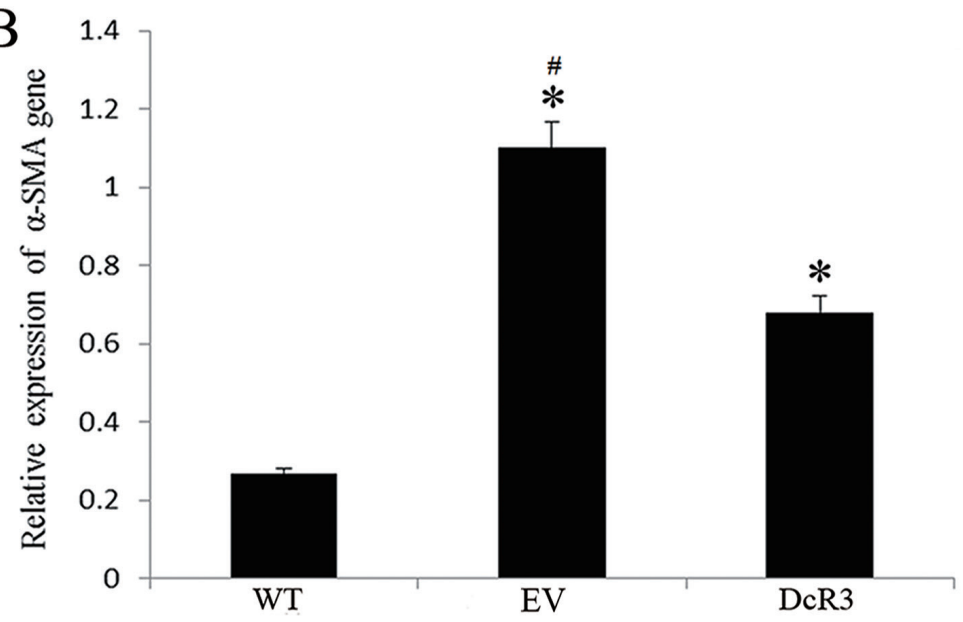

$\mathrm{C}$

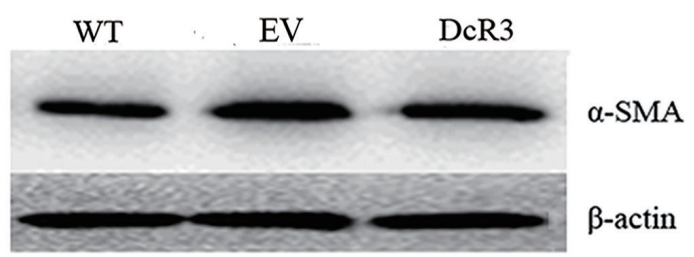

Fig. 3. Histopathological changes of fibrosis and the expression of a-SMA in 1\% DMN-induced rats. A - Masson's trichromic staining of the liver tissue. B - analysis of the a-SMA mRNA expression by qRT-PCR. C - analysis of the a-SMA protein expression by western blot. Data is shown as mean \pm SD of 10 rats in each group

WT - control group (wild-type); EV - 1\% DMN + empty vector group; DcR3 - 1\% DMN + DcR3 vector group; * $p<0.05$ compared with WT group; ${ }^{*} \mathrm{p}<0.05$ compared with EV group; DCR3 - decoy receptor 3; DMN - dimethylnitrosamine. 
A

$\operatorname{DcR3}(\mathrm{H})$
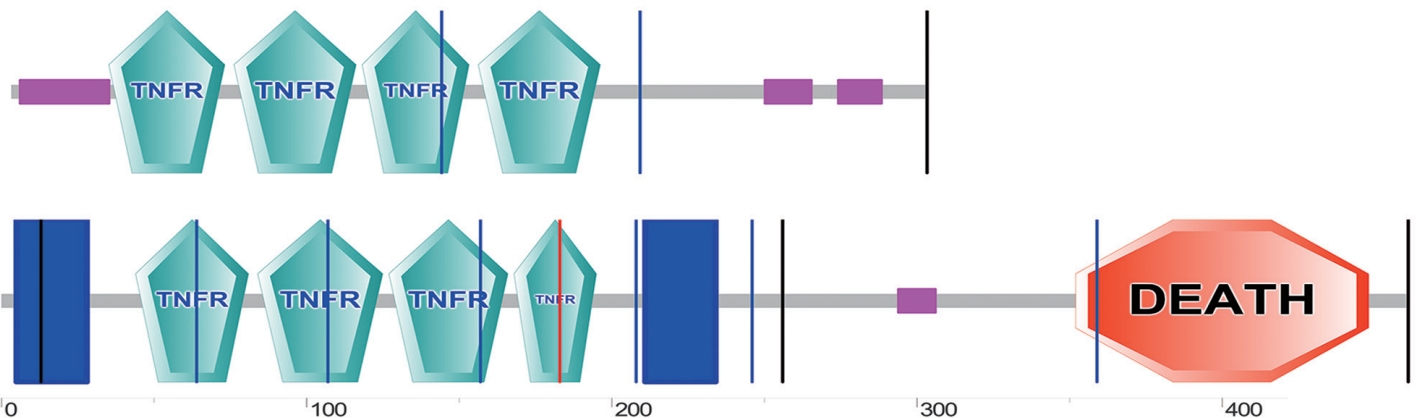

B

$\begin{array}{llll}\text { TNFR1A(H) } 1 & \text { MGLSTVPDLLLPLVLLELLVGIYPSGVIGLVPHLGDREKRDSVCPQGKYIHPQNNSICCTKCHKGTYLYNDCPGPGQDTD } & \text { 80 } \\ \text { TNFR1A(R) } & 1 & \text { MGLPIVPGLLLSLVLLALLMGIHPSGVTGLVPSLGDREKRDNLCPQGKYAHPKNNSICCTKCHKGTYLVSDCPSPGQETV } & \text { 80 } \\ \text { DcR3(H) } & 1 & \text { MRALEGPGLSLLCLVLALPALLPVPAVRGVA--_---_-ETPTYPWRDAE--TGERLVCAQCPPGTFVQRPCRRDSP-TT } & \text { 69 }\end{array}$

81 CRECESGSFTASENHLRHCLSCSKCRKEMGQVEISSCTVDRDTVCGCRKNQYRHYWSENLFQCFNCSLCLNGTVHLSCQE 160

81 CEVCDKGTFTASQNHVRQCLSCKTCRKEMFQVEISPCKADMDTVCGCKKNQFQRYLSETHFQCVDCSPCFNGTVTIPCKE 160

70 CGPCPPRHYTQFWNYLERCRYCNVLCGE-REEEARACHATHNRACRCRTGFFAH--AGFCLEHASCPP--GAGVIAPGTP 144

161 KQNTVCT-CHAGFFLRENECVSCSNCKKSLECTKLCL PQIENVKGTEDSGTTVLLPLVIFFGLCLLSLLFIGLMYR 235

161 KQNTVCN-CHAGFFLSGNECTPCSHCKKNQECMKLCL PPVANVTNPQDSGTAVLLPLVIFLGLCLLFFICISLLCR 235

145 SQNTQCQPCPPGTFSASSS--SSEQCQPHRNCTALGL[20]PLSTRVPGAEECERAVI-DFVAFQDISIKRLQRLLQALE 237

236 YQR-WKSK LYSIVCGKSTPEKEGELEGTTTKPLAPN--PSFSPTPGFTPTLGFSPVPSSTFTSSST YTPGDCPN 306

236 YPQ-WRPR VYSIICRDSAPVKEVEGEGIVTKPLTPASIPAFSPNPGFNPTLGFSTTPRFSPPVSST[5]FGPSNWHN 313

238 APEgWGPT [6] ALQLKLRRRLTELLGAQDGALLVRLLQAL--RVARMPGLERSVRERFLPVH------- -------- 300

307 FAAPRREVAPPyQGADPILATALASDPIPNPLQKWEDS-AHKPQSLDTDDPATLYAVVENVPPLRWKEFVRRLGLSDHEI 385

314 FVPPVREVVPT-QGADPLLYGSLNPVPIPAPVRKWEDVVAAQPQRLDTADPAMLYAVVDGVPPTRWKEFMRLLGLSEHEI 392

386 DRLELQNGRCLREAQYSMLATWRRRTPRREATLELLGRVLRDMDLLGCLEDIEEALCGPAAIPPAPSLLR 455

393 ERLELQNGRCLREAHYSMLEAWRRRTPRHEATLDVVGRVLCDMNLRGCLENIRETLESPAH-SSTTHLPR 461

Fig. 4. Domain prediction and homology analysis of DCR3. A - the structure of DcR3 and rat TNFR1-a; B - the homology analysis among DcR3, human TNFR1- $a$ and rat TNFR1- $a$

TNFR1-a - tumor necrosis factor receptor 1 alpha; $\mathrm{H}$ - human; R - rat; DcR3 - decoy receptor 3.

\section{Discussion}

Hepatic fibrosis has been evidenced to develop into liver cirrhosis with high morbidity and mortality. Some evidence has suggested that hepatic fibrosis caused by sustaining liver injuries is a reversible process. ${ }^{19}$ A growing number of treatment and therapeutic approaches have been identified and used to prevent hepatic fibrosis..$^{20,21}$ However, there are no useful therapies targeting the primary stimuli of fibrogenesis such as a blockade of proinflammatory pathways. In this study, we demonstrated that the DcR3 gene attenuated hepatic fibrosis by anti-inflammatory effects in rats. The DcR3 gene is a member of TNF superfamily; it is also known as TNFSF6B in humans. The DcR3 protein is similar to the extracellular part of rat TNFR1- $\alpha$, with 4 TNF receptor repeats. However, it lost the trigger functional domain. DcR3 competitively binds with Fas and TL1A, but does not recruit a downstream ligand. This may explain the suppression function of DcR3.
Meanwhile, the DcR3 gene may suppress liver inflammation by suppressing the NF-kB pathway. The hepatic inflammation is a hallmark of fibrosis. ${ }^{22}$ Some studies also reported that TNF- $\alpha$, IL- $1 \beta$ and IL- 6 were associated with many liver diseases such as hepatotoxic-induced liver injures. ${ }^{23}$ A study showed that the anti-fibrosis efficacy could be improved by decreasing the TNF- $\alpha$ level. ${ }^{24}$ In addition, overexpressed DcR3 decreased the TNF- $\alpha$, IL-1 $\beta$ and IL- 6 levels in mice. ${ }^{17}$ Our data showed that cytokines in the liver, including TNF- $\alpha$, IL- $1 \beta$ and IL- 6 , were significantly upregulated in the EV group compared with the WT group, indicating increased inflammatory damage to the liver. However, treatment with DcR3 significantly reduced the TNF- $\alpha$, IL- $1 \beta$ and IL- 6 expression of the liver tissue in $1 \% \mathrm{DMN}$-induced liver fibrosis rats. Combined with histology results, DcR3, as a heterogenous protein, did not cause an immunoreaction in rats. In contrast, it suppressed the inflammatory cytokines expression. These results suggested that DcR3 could alleviate liver fibrosis 
caused by $1 \%$ DMN partly through the suppression of the inflammatory response.

Accumulating evidence has demonstrated that binding of DR3/TL1A is associated with 2 downstream signaling cascades. One results in apoptosis and cell death, the other leads to cell proliferation and activation as well as secretion of cytokines. Ma et al. found that TL1A could promote the expression of inflammatory cytokines, such as IL-6, on fibroblast-like synoviocytes of rheumatoid arthritis (RA) patients by the NF- $\mathrm{kB}$ and JNK signaling pathway. ${ }^{25}$ Huang et al. showed that the DcR3 overexpression mitigated the IAV-induced release of pro-inflammatory cytokines by suppressing the IAV-activated NF- $\mathrm{kB}$ pathway. ${ }^{17}$ In our study, we found that DcR3 decreased the levels of inflammatory cytokines in rats with liver fibrosis. Then, we detected NF- $k B$ activity. As expected, an increase in NF- $\mathrm{kB}$ activity was observed in rats with liver fibrosis when compared with WT rats, while NF- $\mathrm{B}$ activation was inhibited by DcR3, implying that the inhibition of NF-kB activation was tightly involved in the anti-inflammatory action of DcR3. Our studies indicated that DcR3 attenuated hepatic fibrosis severity via the suppression of the NF- $\mathrm{B}$ pathway, which was activated by DR3/TL1A. Nevertheless, further studies are required to define the exact mechanism underlying the anti-inflammatory effects of DcR3.

In addition, we also examined the effects of DcR3 on $1 \%$ DMN-induced liver fibrosis in rats by means of histological examination. Masson's trichromic staining showed that DcR3 significantly inhibited 1\% DMN-induced liver fibrosis in rats (Fig. 3). Furthermore, we also examined the $\alpha$-SMA expression. The $\alpha$-SMA expression markedly activated HSCs, which play a critical role in liver fibrogenesis. ${ }^{26}$ RT-PCR and western blot analyses showed that DcR3 could reduce the $\alpha$-SMA expression in hepatic fibrosis rats. These results confirmed the conclusions presented below. Therefore, our findings provided novel evidence of the protective effect of DcR3 in 1\% DMN-induced hepatic fibrosis rats.

\section{Conclusion}

This study showed that treatment with DcR3 was beneficial in terms of antifibrotic effects on inflammatory cytokines induced by $1 \%$ DMN. The protective effects of DcR3 against hepatic fibrosis may be due to the suppression of the NF-kB pathway, which was activated by the DR3/TL1A signaling pathway. This study suggests that DcR3 could be a possible prophylactic for the prevention or therapy of liver fibrosis in humans.

\section{References}

1. Safer AM, Afzal M, Hanafy N, Mousa S. Green tea extract therapy diminishes hepatic fibrosis mediated by dual exposure to carbon tetrachloride and ethanol: A histopathological study. Experimental and Therapeutic Medicine. 2015;9:787-794.

2. Simpson KJ, Lukacs NW, Colletti L, Strieter RM, Kunkel SL. Cytokines and the liver. J Hepatol. 1997;27:1120-1132.
3. Iredale J. Defining therapeutic targets for liver fibrosis: Exploiting the biology of inflammation and repair. Pharmacol Res. 2008;58:129-136.

4. Imajo K, Kessoku T, Honda Y, et al. Magnetic resonance imaging more accurately classifies steatosis and fibrosis in patients with nonalcoholic fatty liver disease than transient elastography. Gastroenterology. 2016;150:626-637.e7.

5. Chen P, Li J, Huo Y, et al. Orphan nuclear receptor NR4A2 inhibits hepatic stellate cell proliferation through MAPK pathway in liver fibrosis. Peer J. 2015;3:1518.

6. Alcolado R, Arthur MJ, Iredale JP. Pathogenesis of liver fibrosis. Clin Sci (Lond). 1997;92:103-112.

7. Ashkenazi A. Targeting death and decoy receptors of the tumournecrosis factor superfamily. Nat Rev Cancer. 2002;2:420-430.

8. Bai C, Connolly B, Metzker ML, et al. Overexpression of M68/DcR3 in human gastrointestinal tract tumors independent of gene amplification and its location in a four-gene cluster. Proc Natl Acad Sci U SA. 2000;97:1230-1235.

9. Pitti RM, Marsters SA, Lawrence DA, et al. Genomic amplification of a decoy receptor for Fas ligand in lung and colon cancer. Nature. 1998;396:699-703.

10. Migone TS, Zhang J, Luo X, et al. TL1A is a TNF-like ligand for DR3 and TR6/DcR3 and functions as a T cell costimulator. Immunity. 2002;16: 479-492.

11. Yu KY, Kwon B, Ni J, Zhai Y, Ebner R, Kwon BS. A newly identified member of tumor necrosis factor receptor superfamily (TR6) suppresses LIGHT-mediated apoptosis. J Biol Chem. 1999;274:13733-13736.

12. Liu YJ, Shao LH, Zhang J, et al. The combination of decoy receptor 3 and soluble triggering receptor expressed on myeloid cells-1 for the diagnosis of nosocomial bacterial meningitis. Ann Clin Microbiol Antimicrob. 2015;14:17.

13. Shi G, Luo H, Wan X, Salcedo TW, Zhang J, Wu J. Mouse T cells receive costimulatory signals from LIGHT, a TNF family member. Blood. 2002; 100:3279-3286.

14. Liang D, Hou Y, Lou X, Chen H. Decoy receptor 3 improves survival in experimental sepsis by suppressing the inflammatory response and lymphocyte apoptosis. PLoS One. 2015;10:0131680.

15. Huang ZM, Kang JK, Chen CY, et al. Decoy receptor 3 suppresses TLR2-mediated B cell activation by targeting NF-kappaB. J Immunol. 2012;188:5867-5876.

16. Shu M, Huang DD, Hung ZA, Hu XR, Zhang S. Inhibition of MAPK and NF-kappaB signaling pathways alleviate carbon tetrachloride (CCl4)induced liver fibrosis in Toll-like receptor 5 (TLR5) deficiency mice. Biochem Biophys Res Commun. 2016;471:233-239.

17. Huang MT, Chen ST, Wu HY, Chen YJ, Chou TY, Hsieh SL. DcR3 suppresses influenza virus-induced macrophage activation and attenuates pulmonary inflammation and lethality. J Mol Med. 2015;93:1131-1143.

18. Ka SM, Sytwu HK, Chang DM, Hsieh SL, Tsai PY, Chen A. Decoy receptor 3 ameliorates an autoimmune crescentic glomerulonephritis model in mice. J Am Soc Nephrol. 2007;18:2473-2485.

19. Hernandez-Gea V, Friedman SL. Pathogenesis of liver fibrosis. Annu Rev Pathol. 2011;6:425-456.

20. Tacke F, Weiskirchen R. Liver fibrosis - pathogenesis and novel therapeutic approaches. Internist (Berl). 2010;51:21-29 [in German].

21. Gangadharan B, Antrobus R, Chittenden D, et al. New approaches for biomarker discovery: The search for liver fibrosis markers in hepatitis C patients. J Proteome Res. 2011;10:2643-2650.

22. Shin DS, Kim KW, Chung HY, Yoon S, Moon JO. Effect of sinapic acid against carbon tetrachloride-induced acute hepatic injury in rats. Arch Pharm Res. 2013;36:626-633.

23. Gao B, Jeong WI, Tian Z. Liver: An organ with predominant innate immunity. Hepatology. 2008;47:729-736.

24. da Silva KA, Paszcuk AF, Passos GF, et al. Activation of cannabinoid receptors by the pentacyclic triterpene alpha, beta-amyrin inhibits inflammatory and neuropathic persistent pain in mice. Pain. 2011;152: 1872-1887.

25. Ma Z, Wang $B$, Wang $M$, et al. TL1A increased IL- 6 production on fibroblast-like synoviocytes by preferentially activating TNF receptor 2 in rheumatoid arthritis. Cytokine. 2016;83:92-98.

26. Friedman SL. Mechanisms of hepatic fibrogenesis. Gastroenterology. 2008;134:1655-1669. 